Muro de la Investigación, 2021(1), enero-junio

ISSN: 2523-2886

Doi: https://doi.org/10.17162/rmi.v6i1.1438

\title{
Programa "Pukllaspa Ñawinchani" y su eficacia para mejorar la comprensión de lectura de los estudiantes de cuarto grado de Educación Primaria
}

\section{Program "Pukllaspa Nawinchani" and its effectiviness to improve the reading comprehension of students of the 4th grade primary level}

\author{
Nataly Chipana Hurtado ${ }^{1}$ \\ Universidad Peruana Unión, Perú ${ }^{1}$
}

Recibido: 12 de setiembre de 2020

Aceptado: 19 de diciembre de 2020

\section{Resumen}

El objetivo de la presente investigación fue determinar la eficacia del Programa: "Pukllaspa Nawinchani" para mejorar la comprensión de lectura de los estudiantes de cuarto grado de Educación Primaria de la I.E.P. N ${ }^{\circ}$ 38542/Mx-P. de San Rafael, Socos, Huamanga, Ayacucho, 2017. Se usó el diseño pre-experimental, con enfoque cuantitativo. La muestra estuvo conformada por un total de 9 participantes. El recojo de la información fue efectuada a través de un instrumento "Guía de análisis de textos" (pre prueba - post prueba), validado por expertos y para el análisis estadístico se utilizó el programa Statistical Package for Social Sciences (SPSS), versión 24.0 y la hoja de cálculo Excel. Los resultados obtenidos en la prueba "T"de student demostraron que existen diferencias significativas $(\mathrm{p}<0,05)$, entre la aplicación del programa y la comprensión de lectura, dado que, en la pre prueba, el 55,6\% de los estudiantes se encontraban en el nivel inicio, y el 44,4\% en proceso; no obstante, este resultado cambió después de la aplicación del Programa "Pukllaspa Ñawinchani", porque en la post prueba el $22,2 \%$ se ubicó en el nivel proceso, el 77,8\% en el nivel logro y ninguno en el nivel inicio. Lo que es una clara evidencia del valor instructivo del programa aplicado, en aspectos de mejoramiento de la comprensión de lectura.

Palabras clave: Comprensión de lectura, nivel literal, nivel inferencial, nivel críticovalorativo

\begin{abstract}
The aim of this research was determining the effectiviness of the Program "Pukllaspa Nawinchani" to improve the reading comprehension of students of the 4th grade primary level from the E.I.P. N ${ }^{\circ} 38542 / \mathrm{Mx}-\mathrm{P}$. from San Rafael, Socos, Huamanga, Ayacucho, 2017. I have used the design pre-experimental, with a quantitative approach. The sample of this research was comprised of a total of 9 participants. The information collection was carried out through of an instrument "Guide of analysis of texts" (pretest - posttest), validated by experts and for statistical analysis. I used the program
\end{abstract}

${ }^{1}$ Correspondencia al autor natalychipana@upeu.edu.pe 
Statistical Package for Social Sciences (SPSS), version 24.0 and Excel spreadsheet. The result actually achieved in the " $T$ " test of student shown that there are significant differences $(p<0,05)$, between the application of the program and the comprehension of reading, given that, the pretest, 55,6\% of the students were on the start level, and the $44,4 \%$ in progress; however, this result changed after of the application of the Program "Pukllaspa Nawinchani", because in the post test the $22.2 \%$ it was in the progress level, the $77,8 \%$ in the reach level and none at the start level. What is a clear evidence of the instructive value of the program applied, on aspects of improvement of reading comprehension.

Keywords: Comprehension of reading, literal level, inferential level, value-related and critical level

\section{Introducción}

El Perú enfrenta retos múltiples en el sistema educativo, dentro de ellas las dificultades académicas que presentan los estudiantes, siendo una de las competencias y capacidades más importantes el proceso de lectura y comprensión de textos. En el sistema educativo, la lectura es la base fundamental de los estudiantes para el desarrollo de conocimientos de todas las áreas curriculares, actualmente es uno de los problemas que se observa en los resultados nacionales (ECE) e internacionales (PISA). La realidad es que nuestros estudiantes no tienen el nivel óptimo de comprensión de lectura y por esta razón se debe realizar un gran esfuerzo por mejorar esta situación.

Sin duda uno una de las habilidades mentales que todo ser humano aprende desde temprana edad es leer. Desde muy pequeños se nos enseña o aprendemos por modelaje a deletrear, debido que la lectura es la llave del éxito y del conocimiento humano, esto hace que sea una actividad imprescindible; por consiguiente, el ser humano se pasa leyendo toda su vida (Barrientos, 2013). En consecuencia, este proceso no es efectivo, debido que la mayoría de los estudiantes aprende a leer, mas no comprende enteramente el contenido o mensaje de lo que lee (León, 2004).

Por otro lado, es un fenómeno mundial y nuestro país no está exento; al contrario, en uno de los más afectados. Según el Organismo para la Cooperación y el Desarrollo Económico (OCDE), los resultados relativos a la competencia en lectura del ciclo 2000, Finlandia, Canadá, Nueva Zelanda, Australia e Irlanda, se encuentran en las posiciones más altas; por otro lado Perú, Indonesia, y países europeos rezagados en el desarrollo continental como Albania y Macedonia se ubican en los últimos lugares de la escala (OCDE, 2007). Asimismo, según el Programa Internacional de 
Evaluación de Estudiantes (PISA), aplicada cada tres años a más de 60 países, en estudiantes de 15 años, nuestro país evidencia resultados poco satisfactorios en lectura (Salas, 2012).

De la misma manera, el Boletín del Ministerio de Educación (2001), en base a un estudio internacional realizado por el Laboratorio de Medición de la Calidad financiado por el BID, en los países de la región: Argentina, Bolivia, Brasil, Chile, Colombia, Costa Rica, Cuba, Honduras, México, Paraguay, Perú, República Dominicana y Venezuela, se aplicaron más de 54 mil pruebas de matemáticas y lenguaje a los estudiantes del tercero y cuarto grados de primaria; los estudiantes peruanos quedaron en último lugar en Matemáticas y antepenúltimo en Lenguaje, básicamente en comprensión de lectura (Ventura \& Quispe, 2015). Años más tarde, en la evaluación mundial de la prueba PISA (2003), concerniente a competencias lectoras (decodificación, comprensión y juicio crítico), llevada a cabo por la UNESCO, los adolescentes peruanos, ocuparon el último lugar entre 43 países de todo el mundo (Canales, 2008).

La I.E.P. N ${ }^{o}$ 38542/Mx-P. de San Rafael, Socos, Huamanga, Ayacucho, no es ajena a estos problemas, debido que se aprecia que sus estudiantes poseen bajo nivel en comprensión de lectura; es decir, leen, pero no comprenden lo que leen, debido a que lo entienden mayormente a nivel literal, mas no logran hacerlo a nivel inferencial y crítico-valorativo. En consecuencia, los estudiantes no alcanzan a desarrollar capacidades, habilidades y destrezas de análisis e interpretación para realizar inferencias y asumir una posición argumentativa frente al texto que leen. Ahora bien, se sabe que, en general, al niño no le fascina leer, pero, creemos necesaria planificar e implementar métodos, planes y programas de mejora de los aprendizajes, en la que la labor docente y de la propia familia son condiciones claves, utilizando estrategias lúdicas, para motivar y crear hábitos de lectura (Reátegui \& Vásquez, 2014).

Los estudiantes no han consolidado los mecanismos de comprensión de lectura adquiridos en los niveles donde verdaderamente deberían haber aprendido a leer (primero segundo grado de primaria); adolecen de los procesos de adquisición y dominio de distintas estrategias que le permitan acceder a la comunicación escrita en su diversidad social y cultural; padres analfabetos que después de la clase, llevan a sus hijos al trabajo de campo o los dejan cuidando a los animalitos; no poseen conductas de visita a la biblioteca de la escuela, la comunidad carecen de bibliotecas; 
padecen de desequilibrio emocional; tienen baja autoestima; dificultad para comprender lecturas en el nivel inferencial y crítico-valorativo; tienen limitaciones para comprender los mensajes de diversos textos; asimismo dejan de leer y aceptan todo texto por válido, ocupándose en otras cosas insignificantes; mantienen escasez de vocabulario y evidencian un pobre desarrollo de sus capacidades de comunicación escrita y verbal en el idioma español. Esta realidad educativa de San Rafael, Socos, Ayacucho, motivó a realizar esta investigación, a fin de mejorar los niveles: literal, inferencial y crítico-valorativo de la comprensión de lectura en idioma español, asimismo desarrollar un buen desempeño académico de las diversas áreas curriculares y lograr una educación integral de calidad.

\section{Revisión de la literatura}

El Programa Internacional de Evaluación de Estudiantes (PISA 2013), muestra que el 54\% de estudiantes se ubica por debajo del nivel de alfabetización lectora. En efecto, la mayoría de estudiantes que cursan la secundaria no son capaces de comprender lo que leen, y tienen limitadas posibilidades de emplear la lectura como una herramienta de aprendizaje y desarrollo personal (Salas, 2006, citado por Ibáñez 2014). Asimismo, cerca del $20 \%$ de los estudiantes de los países de la OCDE poseen competencias lectoras básicas. En este mismo orden, la diferencia en lectura a favor de las niñas disminuyó 12 puntos en la prueba PISA entre 2009 y 2015, mientras que el rendimiento de los niños mejoró (OCDE, 2016).

Tomado como referencia lo antes expuesto, podemos afirmar que a pesar de los múltiples cambios y acciones que se ha tomado en nuestro país para mejorar la comprensión lectora, el problema sigue latente. Según algunos autores, esto se ocasiona debido que su enseñanza solo se limita al análisis de lecturas e informes de las mismas. Por lo cual, debemos entender que leer no consiste en ojear, buscar palabras desconocidas; requiere concentración, conocimiento de estrategias y éstas no se aprenden por descubrimiento personal, sino a través de la mediación del docente (Tunco, 2016). Asimismo, mucha responsabilidad recae sobre la práctica docente, dado que por muchos años no experimentan cambios significativos en cuanto al qué y cómo enseñar (Secretaría de Educación Pública, 2000).

Del mismo modo, la escuela solo se limita a la enseñanza de la lectura, mediante la presentación de tareas u obligaciones estudiantiles, desconociendo que leer es 
mucho más que poseer un caudal de estrategias; es sobre todo una actividad voluntaria y placentera (García, 2016). En este contexto, muchos estudiantes se sienten desmotivados para leer, debido a que se ha tornado en un proceso mecánico, en consecuentica desarrollan sentimientos de frustración que los desmotivan aún más (Denos, 2015). Sin embargo, la lectura es el medio más importante para adquirir conocimientos y construir nuevos significados, haciendo que la persona que lee pueda ser un lector motivado (Barrientos, 2013). En conclusión, por muchos años la lectura solo se la ha estudiado y entendido como un acto mecánico, pasivo, que decodificaba los signos de un texto, o en mejor de los casos, como un elemental instrumento que transmiten conocimientos o informaciones (Yepes, 2011).

Al respecto, el Ministerio de Educación (2015), sostiene que el acto de leer es mucho más que descifrar o comprender un texto, es poder establecer comunicación con él, para preguntar y hallar respuestas, procesar, analizar, deducir, construir significados desde las experiencias previas. Por lo tanto, la escuela juega un rol más que trascendental en su enseñanza, debido que se contribuye al desarrollo intelectual global de los estudiantes (Calderón, Chuquillanqui, \& Valencia, 2013). No obstante, la enseñanza de la lectura es un tanto compleja, puesto que leer para extraer información de textos científicos, literarios, sociales o pedagógicos se requiere dominar aspectos lingüísticos, tener un amplio universo cultural y estrategias metacognitivas que permitan al lector empoderarse de la lectura (Acosta, 2009).

Solé (1992), manifiesta que el problema radica en que se concibe a la lectura como un proceso aislado, sin embargo necesita interacción entre los conocimientos previos y los nuevos que el lector va a formar. Por su parte, Bamberger (1975), menciona que la lectura trae consigo el aumento de capacidad para aprender en general, sobrepasándose en mucho la mera recepción. Por lo tanto, la comprensión lectora guarda una estrecha relación con los aprendizajes que logran los alumnos en sus centros educativos en sus diferentes modalidades y niveles, en especial en el nivel superior por lo complejo que se hace la lectura (Tunco, 2016).

Entonces, se puede afirmar que el éxito o el fracaso de los estudiantes está íntimamente ligado a sus habilidades para leer (Pérez, 2004). Porque de acuerdo con Fons (1999), citado por Almeida (2012), leer es un proceso activo, en el cual el lector construye el significado del texto, pues el significado que tiene un escrito no es una copia de lo que el autor quiso decir, sino una construcción en la que además 
intervienen conceptos, creencias y experiencias que el lector posee, antes de interpretar el texto. Es decir, su esencia está basada en relacionar, criticar o superar las ideas expresadas; no implica, aceptar tácitamente cualquier proposición (Cordero, 2014), sino consiste en la interacción entre el lector y el texto (Bustamante, 2014), convirtiendo al estudiante en un ser que piensa, actúa, crea y expresa todo lo que siente, en tal sentido no podemos tomar esta actividad como algo liviano (Torres, 2003).

Al respecto, Cubas (2007), argumenta que los estudiantes no comprenden cuando leen debido a que este es un proceso muy complejo, mediante el cual funcionan varias operaciones mentales, en el que se otorga significado a hechos, cosas y fenómenos (Cordero, 2014). En este proceso se requiere de gran concentración, dado que de esta forma se producirá una interacción entre el lector y el texto (Gálvez, 2017). Además, se requiere procesos lingüísticos como los culturales (Jaulis, 2016). Por otro lado, muchos creen que la actividad de leer no se concreta, debido a que vivimos en una sociedad muy ruidosa y plagada de tecnología. Esto dificulta la comprensión, dado que la lectura es una actividad que debe ser realizada solitariamente, silenciosamente y como un hecho personal. No obstante, esta aseveración entra en oposición con el objetivo de nuestras asignaturas, que básicamente buscan que los estudiantes sean capaces de comunicarse en español de manera efectiva, con desenvoltura y autonomía (Acosta, 2009).

\section{Materiales y Métodos}

La investigación es de tipo básica, diseño pre-experimental con enfoque cuantitativo, porque para su desarrollo se utilizó una pre prueba, que sirvió de información para identificar la situación en que se encuentran los estudiantes en la comprensión de lectura. Luego, se aplicó la post prueba que brindó la comparación conveniente. Para la recolección de datos se utilizó dos instrumentos: Una guía de análisis de textos y una lista de cotejo, que fue validado por el juicio de 5 expertos en los criterios de claridad, congruencia, contexto y dominio del constructo, constó de 15 indicadores, 3 dimensiones con sus respectivos ítems: Dimensión 1, nivel literal, con 4 ítems; dimensión 2, nivel inferencial, con 7 ítems; y dimensión 3, nivel crítico, con 4 ítems, de acuerdo con la Operacionalización de las variables. Los niveles de evaluación fueron, inicio (C), proceso (B) y logro (A), aplicados en el proceso de pre 
prueba y post prueba del Programa "Pukllaspa Ñawinchani".

Por ser la muestra menor de 50, se utilizó la prueba de Shapiro - Wilk, y para el análisis estadístico, por ser de datos paramétricos en la pre prueba y post prueba, se aplicó el estadístico de la prueba $\mathrm{T}$ de student. Todo el cálculo estadístico se dio a través del software Statistical Package for the Social Sciences (SPSS), versión 24.0 y la hoja de cálculo Excel Los análisis se realizaron con un 95\% de confianza y un 5\% de significancia de error.

\section{Resultados}

Los resultados que se evidencian en la tabla 1 , muestran que antes de la aplicación del programa, en la evaluación de la pre prueba, el 55,6\% de los estudiantes se encontraba en el nivel inicio, mientras que el $44,4 \%$ se ubicó en el nivel proceso; este resultado cambió después de la ejecución del Programa “Pukllaspa Nawinchani”, debido a que en la evaluación de la post prueba los resultados mostraron que el 22,2\% de los estudiantes se encontraba en el nivel proceso y el 77,8\% alcanzó el nivel logro. Este último no aparecía en los resultados de pre prueba, lo que significa que el programa educativo aplicado es eficaz, académicamente hablando.

Tabla 1

Nivel de evaluación de la comprensión de lectura en la pre prueba y post prueba

\begin{tabular}{lccccc}
\hline \multirow{2}{*}{ Niveles } & \multicolumn{2}{c}{ Antes } & & \multicolumn{2}{c}{ Después } \\
\cline { 2 - 3 } \cline { 5 - 6 } & Frecuencia & Porcentaje & & Frecuencia & Porcentaje \\
\hline Inicio & 5 & $55,6 \%$ & & 0 & $0,0 \%$ \\
Proceso & 4 & $44,4 \%$ & & 2 & $22,2 \%$ \\
Logro & 0 & $0,0 \%$ & & 7 & $77,8 \%$ \\
Total & $\mathbf{9}$ & $\mathbf{1 0 0 , 0 \%}$ & & $\mathbf{9}$ & $\mathbf{1 0 0 , 0 \%}$ \\
\hline
\end{tabular}

Respecto a la dimensión literal, la tabla 2 evidencia que antes de la aplicación del programa, el 22,2\% de los estudiantes se encontraba en el nivel inicio, el 33,3\% en el nivel proceso y el 44,5\% en el nivel logro; estos resultados cambiaron después de la ejecución del Programa, debido a que, en la evaluación de la post prueba, el 100\% de los estudiantes alcanzó el nivel logro. Este hecho permite concluir que el Programa "Pukllaspa Ñawinchani” es significativamente eficaz para la enseñanza de la comprensión de lectura, sobre todo en los aspectos literales de la comprensión de lectura 
de los estudiantes de educación primaria de San Rafael, Socos, Ayacucho.

Tabla 2

Nivel de comprensión de lectura, dimensión literal, en la pre prueba y post prueba

\begin{tabular}{lcccc}
\hline \multirow{2}{*}{ Niveles } & \multicolumn{2}{c}{ Antes } & & \multicolumn{2}{c}{ Después } \\
\cline { 2 - 5 } \cline { 4 - 5 } & \multirow{2}{*}{ Frecuencia } & Porcentaje & Frecuencia & Porcentaje \\
\hline Inicio & 2 & $22,2 \%$ & 0 & $0,0 \%$ \\
Proceso & 3 & $33,3 \%$ & 0 & $0,0 \%$ \\
Logro & 4 & $44,5 \%$ & 9 & $100,0 \%$ \\
Total & $\mathbf{9}$ & $\mathbf{1 0 0 , 0 \%}$ & $\mathbf{9}$ & $\mathbf{1 0 0 , 0 \%}$ \\
\hline
\end{tabular}

Respecto a la dimensión inferencial, los resultados de la tabla 3, revelan que, antes de la aplicación del programa, el 77,8\% de los estudiantes se encontraba en el nivel inicio, mientras que el $22,2 \%$ en el nivel proceso; estos resultados cambiaron después de la ejecución del Programa "Pukllaspa Ñawinchani”, porque, en la evaluación de la post prueba, los resultados mostraron que el 22,2\% de los estudiantes se encontraba en el nivel proceso y el 77,8\% en el nivel logro. Lo que muestra que la comprensión inferencial de la lectura mejora significativamente cuando se implementa dicho programa educativo.

Tabla 3

Nivel de comprensión de lectura, dimensión inferencial, en la pre prueba y post prueba

\begin{tabular}{lccccc}
\hline \multirow{2}{*}{ Niveles } & \multicolumn{2}{c}{ Antes } & & \multicolumn{2}{c}{ Después } \\
\cline { 2 - 3 } \cline { 6 - 6 } & Frecuencia & Porcentaje & & Frecuencia & Porcentaje \\
\hline Inicio & 7 & $77,8 \%$ & & 0 & $0,0 \%$ \\
Proceso & 2 & $22,2 \%$ & & 2 & $22,2 \%$ \\
Logro & 0 & $0,0 \%$ & & 7 & $77,8 \%$ \\
Total & $\mathbf{9}$ & $\mathbf{1 0 0 , 0 \%}$ & & $\mathbf{9}$ & $\mathbf{1 0 0 , 0 \%}$ \\
\hline
\end{tabular}

Finalmente, en la dimensión critico-valorativo, la tabla 4 muestra que antes de la aplicación del programa, el 100\% de los estudiantes se encontraba en el nivel inicio; este resultado cambió después de la ejecución del Programa “Pukllaspa Nawinchani”; 
porque, en la evaluación de la post prueba, el 22,2\% de los estudiantes se encontraba en el nivel proceso y el 77,8\%, en el nivel logro. Como se ve, en los resultados de post prueba, el programa muestra una significativa eficacia en la comprensión críticovalorativa de la lectura.

Tabla 4

Nivel de comprensión de lectura, dimensión crítico-valorativo, en la pre prueba y post prueba

\begin{tabular}{lcccc}
\hline \multirow{2}{*}{ Niveles } & \multicolumn{2}{c}{ Antes } & & \multicolumn{2}{c}{ Después } \\
\cline { 2 - 5 } & Frecuencia & Porcentaje & Frecuencia & Porcentaje \\
\hline Inicio & 9 & $100,0 \%$ & 0 & $0,0 \%$ \\
Proceso & 0 & $0,0 \%$ & 2 & $22,2 \%$ \\
Logro & 0 & $0,0 \%$ & 7 & $77,8 \%$ \\
\hline Total & $\mathbf{9}$ & $\mathbf{1 0 0 , 0 \%}$ & $\mathbf{9}$ & $\mathbf{1 0 0 , 0 \%}$ \\
\hline
\end{tabular}

\section{Discusión de resultados}

El propósito de esta investigación fue determinar la eficacia del Programa “Pukllaspa Nawinchani” para mejorar la comprensión de lectura de los estudiantes de cuarto grado de Educación Primaria de la I.E.P. $N^{\circ}$ 38542/Mx-P. de San Rafael, Socos, Humanga, Ayacucho; en ese sentido, el estudio describe los niveles de comprensión de lectura en el pre y post prueba de un solo grupo. Con estos resultados queda validado y cumplido dicho objetivo, confirmándose la efectividad del Programa “Pukllaspa Ñawinchani”. Para el análisis de este estudio se introdujo datos cuantitativos sustituidos por los puntajes en las pruebas (pre-post) que permitió verificar los resultados de mejora significativa en las dimensiones (niveles): literal, inferencial y crítico-valorativo de la comprensión de lectura., con distintos autores que investigaron sobre el mismo problema.

Los resultados de la dimensión literal, en la cual se consideró una comprensión de lectura básica, decodificación de palabras, oraciones, párrafos, obtención de la información, construcción superficialmente en el texto, es decir se buscó reproducir literalmente lo que dice el texto; los resultados indican que el Programa "Pukllaspa Ñawinchani" es significativamente eficaz. El 100\% de estudiantes obtuvo el nivel logro de le evaluación, en comprensión literal de lectura. Esto es semejante con los 
estudios obtenidos por Jaulis (2016), quien, en el mismo nivel, obtuvo el 90,0\% de mejora. Lo que indica que el Programa "Pukllaspa Ñawinchani", aplicado por la investigadora, tuvo mejores resultados. La diferencia no necesariamente implica invalidar una u otra investigación realizada, sino de integrar y hasta de complementar métodos, estrategias y actitudes aplicados en los procesos áulicos. Este hecho permite concluir que el Programa "Pukllaspa Ñawinchani” es significativamente eficaz para la enseñanza de la comprensión de lectura, sobre todo en los aspectos literales de la comprensión de lectura de los estudiantes de educación primaria de San Rafael, Socos.

En la dimensión inferencial, lo cual incluyó la intuición y experiencia personal como base para conjeturas e hipótesis, es decir integrar pensamiento e imaginación con el propósito de obtener información y establecer conclusiones, para esto fue necesario que los estudiantes utilicen sus saberes previos y reconozcan el lenguaje figurado; los resultados obtenidos con la aplicación del Programa "Pukllaspa Ñawinchani", se logró el 77,8\% de efectividad en el nivel logro de la evaluación de los niños de educación primaria. Lo que tiene concordancia con las obtenidas por Velarde (2018), quien, en la dimensión inferencial, obtuvo resultados del 100\% de efectividad. La diferencia de los resultados se debe a los niveles en que se encontraban ambos grupos antes de la aplicación del programa, ya que los participantes del programa de Velarde el 25,0\% se encontraron en el nivel proceso, mientras los datos de la investigadora del presente trabajo fueron que el $77,8 \%$ se encontraron en el nivel inicio.

En lo que concierne a la dimensión crítico-valorativo, que implica comprensión global el texto, reconocimiento de las intenciones del autor, superestructuralidad del texto, postura frente a lo que dice el texto e integrándolo con lo que se sabe y es capaz de resumirlo. Los resultados que se obtuvieron en esta investigación fueron eficaces, ya que el 77,8\% de los estudiantes se ubicaron en el niel logro de la evaluación de los niños de primaria. Estos datos coinciden claramente con informes de otros investigadores, uno de ellos Álvarez (2017), quien obtuvo un 88,46\% de efectividad. La diferencia de los resultados se debe a que los estudiantes de San Rafael, en la pre prueba, se encontraban en un $100 \%$ en el nivel inicio de la evaluación. Esta evidencia justifica y proporciona méritos valiosos al Programa "Pukllaspa Ñawinchani” para la comprensión de lectura. 


\section{Conclusiones}

El objetivo de este trabajo de investigación fue evidenciar la efectividad del Programa "Pukllaspa Nawinchani”, en la mejora de la comprensión de lectura de los estudiantes de cuarto grado de Educación Primaria de la I.E.P. N ${ }^{o}$ 38542/Mx-P. de San Rafael, Socos, Huamanga, Ayacucho, 2017, y las conclusiones son como sigue:

El Programa "Pukllaspa Ñawinchani”, es eficaz para mejorar la comprensión de lectura de los estudiantes de cuarto grado de Educación Primaria de la I.E.P. $\mathrm{N}^{\mathrm{o}}$ 38542/Mx-P. de San Rafael, Socos, Huamanga, Ayacucho, 2017, debido a los resultados obtenidos a través la prueba $\mathrm{t}$ de student para muestras relacionadas evidencian el valor $\mathrm{t}$ cal $=19.453>\mathrm{t}$ crít $=1.859$ y un nivel de significancia $\mathrm{p}=0.000$ $<0.05$, asimismo, en la evaluación de la pre prueba sobre la comprensión de lectura, el $55,6 \%$ de los estudiantes se encontraban en el nivel inicio, mientras que el $44,4 \%$ se ubicaron en el nivel proceso; este resultado tuvo una variación luego de la aplicación del programa porque en la post prueba el $22,2 \%$ de los estudiantes se encontró en el nivel proceso, mientras que el $77,8 \%$ se ubicaron en el nivel logro. Como se ve, los resultados de la aplicación del Programa "Pukllaspa Nawinchani” es altamente eficaz en el proceso enseñanza aprendizaje de la comprensión de lectura para los estudiantes de cuarto grado de Educación Primaria, especialmente de las zonas bilingües como el de la región quechua español en el Perú.

El Programa "Pukllaspa Ñawinchani”, es significativamente eficaz en la mejora de la comprensión de lectura en el nivel literal de los estudiantes de cuarto grado de Educación Primaria de la I.E.P. N ${ }^{\circ}$ 38542/Mx-P. de San Rafael, Socos, Huamanga, Ayacucho, 2017, debido a la prueba de rangos con signo de Wilcoxon para muestras relacionadas muestra un valor $Z=-2.701 \mathrm{y}$ un nivel de significancia $\mathrm{p}=0.007<0.05$, asimismo, en la evaluación de la pre prueba sobre la comprensión de lectura de nivel literal, el 22,2\% de los estudiantes se encontraron en el nivel inicio, el 33,3\% en el nivel proceso y el 44,5\% en el nivel logro; este resultado tuvo una variación luego de la aplicación del programa porque en la post prueba el 100\% de los estudiantes alcanzó el nivel logro.

El Programa "Pukllaspa Ñawinchani”, es eficaz en la mejora de la comprensión de lectura en el nivel inferencial de los estudiantes de cuarto grado de Educación Primaria de la I.E.P. No 38542/Mx-P. de San Rafael, Socos, Huamanga, Ayacucho, 
2017, debido a los resultados obtenidos a través la prueba $t$ de student para muestras relacionadas que evidencian el valor $\mathrm{t}$ cal $=13.993>\mathrm{t}$ crít $=1.859 \mathrm{y}$ un nivel de significancia $\mathrm{p}=0.000<0.05$, asimismo, en la evaluación de la pre prueba sobre la comprensión de lectura de nivel inferencial, el 77,8\% de los estudiantes se encontraban en el nivel inicio, mientras que el 22,2\% se ubicó en el nivel proceso; este resultado tuvo una variación luego de la aplicación del programa, porque en la post prueba el $22,2 \%$ de los estudiantes se encontró en el nivel proceso, mientras que el 77,8\% se ubicó en el nivel logro.

El Programa "Pukllaspa Ñawinchani”, es eficaz en la mejora de la comprensión de lectura en el nivel crítico-valorativo de los estudiantes de cuarto grado de Educación Primaria de la I.E.P. No 38542/Mx-P. de San Rafael, Socos, Huamanga, Ayacucho, 2017, debido a la prueba de rangos con signo de Wilcoxon para muestras relacionadas que muestra un valor $Z=-2.751$ y un nivel de significancia $p=0.006<0.05$ asimismo, en la evaluación de la pre prueba sobre la comprensión de lectura de nivel críticovalorativo, el 100\% de los estudiantes se encontró en el nivel inicio; este resultado tuvo una variación luego de la aplicación del programa, porque en la post prueba el 22,2\% de los estudiantes se encontró en el nivel proceso, mientras que el 77,8\% se ubicó en el nivel logro.

\section{Referencias}

Acosta, I. (2009). La comprensión lectora, enfoques y estrategias utilizadas durante el proceso de aprendizaje del idioma español como segunda lengua. Universidad de Granada. Recuperado a partir de https://hera.ugr.es/tesisugr/1870914x.pdf

Almeida, H. B. (2012). Aplicaciòn de estrategias cognitivas y metacognitivas para desarrollar la comprensiòn lectora en niños de 5to año de EGB de la Escuela Fiscal mixta Selfina Castro, parroquia Alòag, Cantòn Mejìa, Provincia de Pichinta. Ambato. Universida de Salamanca, España.

Alvarez, M. (2017). Efectos del programa las estrategias del buen lector en la comprensión lectora en estudiantes de educación secundaria- San Miguel 2017. Universidad César Vallejo.

Bamberger, R. (1975). La promoción de la lectura. Recuperado a partir de http://unesdoc.unesco.org/images/0013/001343/134347so.pdf

Barrientos, M. I. (2013). «Influencia del programa de estrategias para desarrollar la comprensión lectora en niños de 4o. grado del colegio Capoulliez». Universidad Rafael Landìvar.

Bustamante, M. (2014). Programa de habilidades comunicativas para mejorar el nivel 
de cmprensiòn lectora en los alumnos de 5 to de secundaria del cntro educativo particular «Juan Pablo II Trujillo». Universidad Privada Antenor Orrego. Recuperado a partir de http://repositorio.upao.edu.pe/bitstream/upaorep/932/1/BUSTAMANTE_MA RIELLA_HABILIDADES_COMUNICATIVAS_COMPRENSIÓN.pdf

Calderón, M. M., Chuquillanqui, R. M., \& Valencia, L. A. (2013). Las estrategias para la comprensión de textos y los niveles de comprensión lectora en los estudiantes del 4to grado de primaria en la i.e. no 0058, Ugel 06, Lurigancho- Chosica, 2013. Universidad Nacional de Educación Enrique Guzmán y Valle. Recuperado a partir de http://repositorio.une.edu.pe/bitstream/handle/UNE/792/TS PC-Ep $\quad$ C21 2013.pdf? sequence $=1 \&$ isAllowed $=y$

Canales, R. (2008). Procesos cognitivos y estrategias psicolingüísticas que intervienen en la lectura comprensiva: Diseño y ejecución de un programa experimental en niños con problemas de aprendizaje. Revista IIPSI, 11(1), 81-100. Recuperado a partir http://revistasinvestigacion.unmsm.edu.pe/index.php/psico/article/view/3878

Cordero, A. (2014). Mejora en los niveles de comprensión lectora de los alumnos del sexto grado de educación primaria de la institución educativa «San Ramón» provincia de Huamanga - Ayacucho mediante estrategias de lecturas - 2013. Universidad Catótica los Ángeles Chimbote. Recuperado a partir de http://repositorio.uladech.edu.pe/bitstream/handle/123456789/1533/COMPRE NSION_LECTORA_ESTRATEGIAS_DE_LECTURA_CORDERO_HUAM ANI_ARTEMIO.pdf?sequence $=1 \&$ is Allowed $=\mathrm{y}$

Cubas, A. C. (2007). Actitudes hacia la lectura y niveles de comprension lectora en estudiantes de sexto grado de primaria. Pontificia Univerdidad Catòlica del Perù. Recuperado a partir de http://tesis.pucp.edu.pe/repositorio/bitstream/handle/123456789/427/CUBAS _BARRUETO_ANA_ACTITUDES_HACIA_LECTURA.pdf?sequence=1

Denos, F. (2015). Actitud para leer textos en inglés y la comprensión lectora en los estudiantes del tercer grado del nivel secundario de la institución educativa mixta «Bernardo Tambohuacso» del distrito de Pisac - provincia de calca región cusco - 2015. Universidad nacional San Agustín de Arequipa.

Gálvez, F. M. (2017). Estrategias didácticas para mejorar la comprensión lectora en los estudiantes de segundo grado de la institución educativa $\mathrm{n}^{\circ} 82260$ del distrito de san Bernardino, provincia de san pablo - región Cajamarca 2015. Universidad Nacional Pedro Ruiz Gallo.

García, M. I. (2016). Comprensión lectora y resolución de problemas matemáticos en los alumnos del segundo grado de educación primaria de una institución educativa privada del distrito de Santiago de surco perteneciente a la Ugel 07. Universidad Ricardo Palma.

Ibáñez, K. M. (2014). Estrategia de aprendizaje SQ4R para mejorar los niveles de comprensión lectora en el idioma inglés de los alumnos del ciclo básico III del Instituto Peruano Americano «El Cultural» de la ciudad de Trujillo, 2014. Universidad Privada Antenor Orrego - UPAO. Recuperado a partir de http://repositorio.upao.edu.pe/bitstream/upaorep/2299/2/RE_MAESTRIA_ED 


\section{U\%282\%29_KATHERIN.IBAÑEZ_ESTRATEGIA.DE.APRENDIZAJE.SQ 4R.PARA.MEJORAR.LA.COMPRENSION.LECTORA_DATOS.pdf}

Jaulis, I. C. (2016). Efectividad del programa «Leo y comprendo» para la comprensión de lectura, en estudiantes del segundo grado del nivel primario de la Institución Educativa Adventista Tercer Milenio de Pucallpa. Universidad Peruana Unión. Recuperado a partir de http://repositorio.upeu.edu.pe/bitstream/handle/UPEU/538/Jocabeth_Tesis_ba chiller_2016.pdf?sequence $=1$

León, J. A. (2004). ¿Por qué las personas no comprenden lo que leen? (2004). Nebrija, 7, 10. Recuperado a partir de http://www.nebrija.com/revistalinguistica/files/articulosPDF/articulo_530df80e7700c.pdf

OCDE. (2007). El programa PISA de la OCDE. Que es y para que sirve. Recuperado a partir de http://www.oecd.org/pisa/39730818.pdf

OCDE. (2016). Pisa 2015 Resultados Clave. Organización para la Cooperación y el Desarrollo Económicos. https://doi.org/http://dx.doi.org/10.1787/9789264266490-en

Pèrez, Y. (2004). Uso de estrategias para mejorar el nivel de comprensión lectora en los niños De $4^{\circ}$ grado de educación básica de la U.E. «Tomás Rafael Giménez» de Barquisimeto. Universidad Nacional Abierta. Recuperado a partir de http://onlinelibrary.wiley.com/doi/10.1002/cbdv.200490137/abstract

Reátegui, L., \& Vásquez, L. (2014). Factores que influyen en el hábito de lectura en estudiantes del 5to. Grado de primaria de las instituciones educativas públicas de Punchana - 2014. Universidad Nacional de la Amazonia Peruana Facultad de Ciencias de la Educación y Humanidades. Recuperado a partir de http://repositorio.unapiquitos.edu.pe/bitstream/handle/UNAP/3504/Luisa_Tes is_Titulo_2014.pdf?sequence $=1$ \&isAllowed $=\mathrm{y}$

Salas, P. (2012). El desarrollo de la comprensión lectora en los estudiantes del tercer semestre del nivel medio superior de la universidad autónoma de nuevo león. Universidad Autónoma de Nuevo León Facultad de Filosofía y Letras División de Estudios de Posgrado. Recuperado a partir de http://eprints.uanl.mx/3230/1/1080256466.pdf

Secretaría de Educación Pública. (2000). Libro para el maestro (1 ed.). México. Recuperado a partir de http://pep.ieepo.oaxaca.gob.mx/recursos/libro electronico/cuarto/docente/LPM-ESPANOL-4-P-001-108.pdf

Solé, I. (1992). Estrategias de comprensión de la lectura. Cuadernos de Pedagogía, 216, 25-27. Recuperado a partir de http://www.lecturayvida.fahce.unlp.edu.ar/

Torres, M. E. (2003). La lectura. Factores y actividades que enriquecen el proceso. Educere, 6(20), 389-396. Recuperado a partir de http://www.redalyc.org/articulo.oa?id=35662006

Tunco, E. M. (2016). Asociación de los niveles de comprensión lectora y aprendizajes esperados en el área de comunicación de los estudiantes del tercer grado de la institución educativa primaria «Jorge Chávez» Juliaca, 2016. Universidad Peruana Unión. Recuperado a partir de http://www.upeu.edu.pe/mision-yvision/ 
Velarde, K. (2018). Aplicación del programa «Allinta yachanapaq ñawinchasun» para mejorar la comprensión de lectura, en los estudiantes quechua-hablantes de primero y segundo grado del aula multigrado de educación primaria de la I.E. $\mathrm{N}^{\circ} 38542$ San Rafael, Socos, Huamanga, Ayacucho, 2017. Universidad Peruana Unión.

Ventura, A., \& Quispe, A. (2015). El aprendizaje cooperativo y su influencia en la comprensión lectora en estudiantes del segundo grado «B»de educación secundaria, planteles de aplicación «Guamán Poma de Ayala»., 196.

Yepes, M. C. (2011). Nivel de comprensión lectora en los estudiantes del quinto grado según tipo de institución educativa estatal y particular. Universidad San Ignacio De Loyola. Recuperado a partir de http://repositorio.usil.edu.pe/bitstream/123456789/1353/1/2011_Yepes_Nivel de comprensión lectora en los estudiantes del quinto grado según tipo de institución educativa estatal y particular.pdf 